\title{
HYBRID BONE SCINTIGRAPHY IN GASTROINTESTINAL MALIGNANCIES - INSTITUTIONAL EXPERIENCE
}

\author{
Nazia Rashid ${ }^{1}$, Saima Riaz ${ }^{1}$, Humayun Bashir ${ }^{1}$, Shafqat Mehmood ${ }^{2}$ \\ ${ }^{1}$ Department Nuclear Medicine, Shaukat Khanum Memorial Cancer Hospital and Research Centre, Lahore, \\ Pakistan, ${ }^{2}$ Department Internal Medicine, Shaukat Khanum Memorial Cancer Hospital and Research Centre, \\ Lahore, Pakistan \\ Received: 28 February 2018 / Accepted: 21 March 2018
}

\begin{abstract}
Objective: Bone metastases in gastrointestinal (GI) malignancies are uncommon and known to be predominantly lytic thus rendering a limited role for conventional bone scintigraphy. The aim of the study is to review Tc99m MDP bone scans performed in patients with known GI malignancy and the utility of Single-photon emission computed tomography (SPECT)/CT in characterization of bone lesions.

Methods: Retrospective review of bone scans was done from June 2014 to December 2016 in patients with known GI malignancy, using electronic Hospital Information System.

Results: A total of 110 patients (60 males, 50 females; Age range: 22-84 years, Mean Age 51.4 years with SD of 14.8) had bone scan over a period of 30 months. The commonest indications for referral were suspected bone metastasis on other imaging modalities including [CT $(n=56)$, Magnetic resonance imaging $(n=10)$, Positron emission tomography/CT $(n=6)]$, musculoskeletal pain $(n=37)$, pathological fracture $(n=1)$, neurological symptoms $(n=1)$, hypercalcemia $(n=1)$ and others $(n=14)$ including restaging workup etc. Metastatic lesions were identified in $32(29 \%)$ patients whereas $78(71 \%)$ patients had benign non-aggressive lesions leading to normal bone scans. Among 32 patients with osseous metastasis, $8(25 \%)$ patients had unifocal lesion; axial skeleton $(n=2)$ appendicular skeleton $(n=6), 24(75 \%)$ patients had multifocal lesions; axial skeleton $(n=6)$, appendicular skeleton $(n=6)$ and both axial + appendicular $(n=12)$. Four $(12 \%)$ patients had concurrent visceral metastases. In our cohort, based on the location of primary tumour, the frequencies of osseous metastasis were; esophagus $=15$ out of $43(35 \%)$, gastric $=7$ out of $18(39 \%)$, gastro-esophageal junction $=1$ out of $8(1.5 \%)$ and colorectal $=9$ out of $40(22.5)$. SPECT/CT was acquired in 29 out of 110 patients, which characterized metastatic lesions $(n=12)$ and benign looking non-aggressive entities $(n=17)$. Overall, bone scan upstaged disease in $31 \%$ and down staged $15 \%$ patients.
\end{abstract}

Conclusion: Bone metastases in GI malignancies, though uncommon, show an aggressive pattern, with axial and appendicular involvement, and can be readily identified with hybrid bone scintigraphy in symptomatic patients.

Key words: Hybrid imaging, Tc99m MDP, bone metastases, gastrointestinal malignancies

\section{Introduction}

Cancer was established to be the second leading cause of mortality in 2015. The incidence of cancer is expected to incline in future on the basis of epidemiological transitions. Therefore, we are at imminent need for early cancer diagnosis and early treatment along with

Correspondence: Dr. Nazia Rashid, Department Nuclear Medicine, Shaukat Kahnum Memorial Cancer Hospital and Research Centre,

Lahore, Pakistan. Email: nazia5809@gmail.com preventive methods to decrease the rate of death in upcoming years. ${ }^{[1]}$

Gastrointestinal (GI) tract malignancies are one of the most incident cancers. However, the incidence of skeletal metastases in GI tumour is not very high. Once the cancer spread to the bones, it has significant implication and impact on patient management, outcome and prognosis. The reported incidence of skeletal metastases from GI tumours ranges from $3 \%$ to $5 \%{ }^{[2]}$ 
Bone metastasis increases the morbidity and alters the quality of life. Life events related to bone metastasis are referred to as skeletal-related events (SREs). SREs include bone pains, spinal-cord compression, pathological fractures and hypercalcemia etc. ${ }^{[2]}$ Pathological fractures due to metastatic skeletal disease increases the risk of death.

Therefore utmost goal of therapy in disseminated malignancies is to prevent pathological fractures. ${ }^{[3]}$

Regarding treatment, the aim is always to prevent SREs and multimodality comes into play at this stage. Targeted local radiotherapeutics can be used for limited bone disease or localized skeletal metastasis. However, in multifocal metastatic cases chemotherapy or hormonal therapies are of benefit. ${ }^{[4]}$

Primary presentation of many GI tumours can be bony metastases without prior GI symptoms. It is rare and infrequently reported in the recent literature. ${ }^{[5]}$

Therefore morbidity related to SREs needs to be promptly addressed to detour the devastating results of bone metastases. ${ }^{[6]}$

In the current guidelines, no screening investigation is in practice to evaluate the bone metastasis exclusively at baseline staging work up. Bone metastases in GI malignancies are predominantly osteolytic, thus rendering a limited role for conventional bone scintigraphy. Therefore, bone scintigraphy is done for specific clinical indication or for suspicion of bone metastases raised on imaging. ${ }^{[2]}$

Single-photon emission computed tomography (SPECT)/ CT in addition to bone scintigraphy increases the diagnostic performance and accuracy in terms of cancers staging. ${ }^{[7]}$

\section{Purpose}

The aim of this study is to review Tc99m MDP bone scans performed in patients with known GI malignancy and the utility of SPECT/CT to characterize the bone lesions.

\section{Methods}

Electronic medical records of 110 consecutive patients with GI malignancies were reviewed. Retrospective review of bone scans was done using electronic Hospital Information System, acquired between June 2014 and December 2016. All patients underwent Tc99m MDP bone scan with dosage according to weight based criteria. Where suspicious, the osseous uptake on Tc-99m MDP bone scan was further correlated with additional radiological imaging or SPECT/CT in a few cases.

The association of bone metastases with gender and age was evaluated. Furthermore lesions were evaluated for uni or multifocality.

Finally, the change in the stage of the tumour was assessed on the basis of bone scan results.

\section{Results}

A total of 110 patients (60 males, 50 females; age range: 22-84 years) had bone scan over a 30 months period.

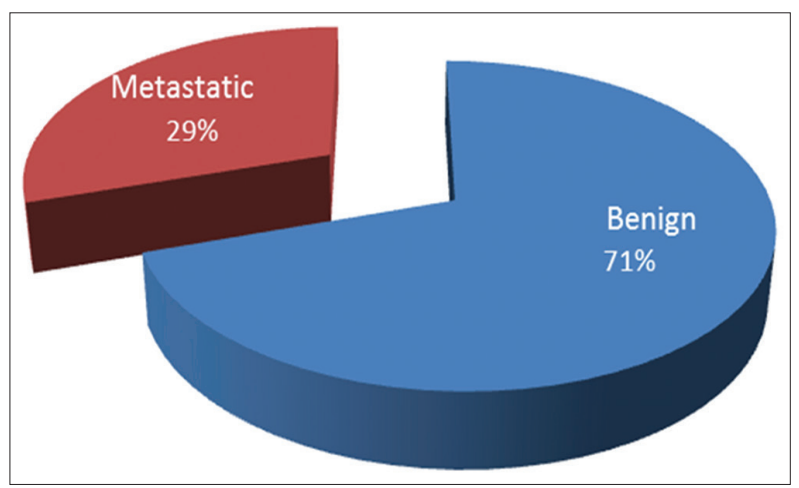

Figure 1: Characterization of skeletal lesions

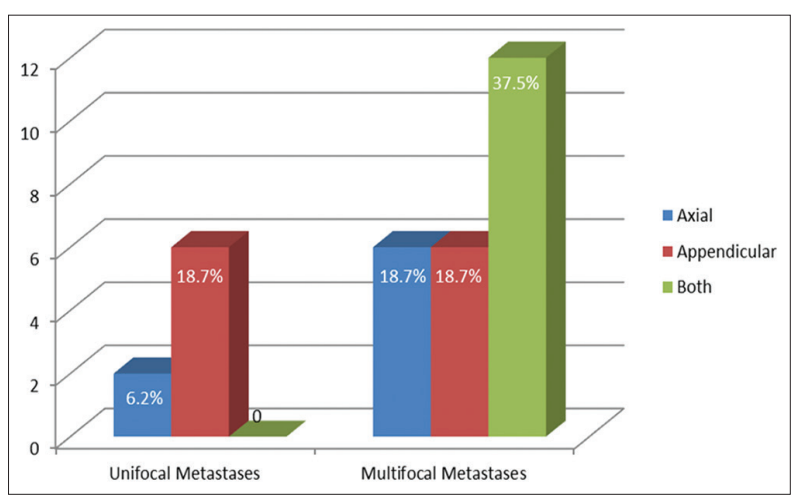

Figure 2: Distribution of skeletal metastases in axial and appendicular skeleton 


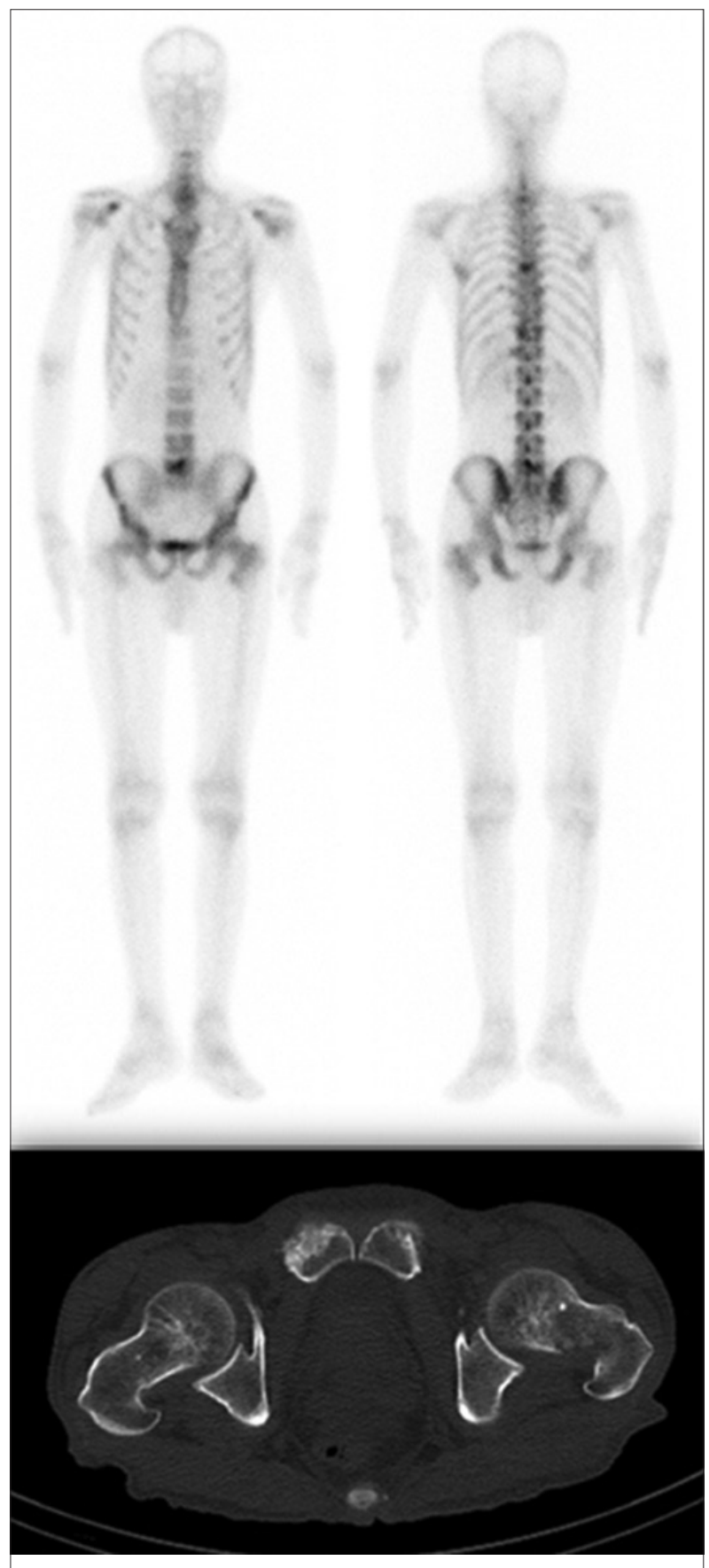

Figure 3: 62-year-old male with Squamous cell carcinoma esophagus, on follow-up computed tomography suspicion of metastases was raised, Bone Scan showed multiple lesions and upgraded the stage by involvement of left femoral neck lytic lesion

The common indications for referral were suspected bone metastasis on other imaging modalities including [CT $(n=56)$, Magnetic resonance imaging (MRI)

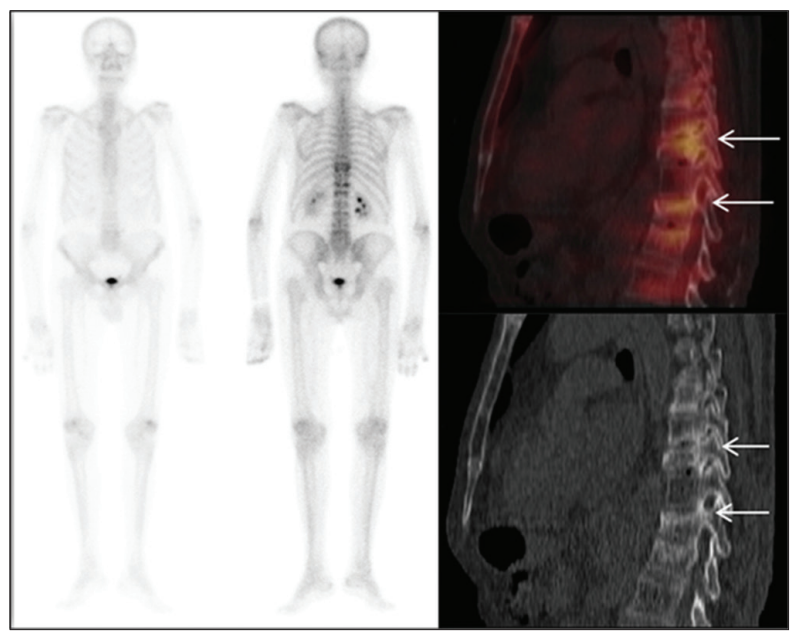

Figure 4: 74-year-male with squamous cell carcinoma esophagus, status/post radiation therapy. Patient developed backache and underwent bone scan. Scan showed metastases Single-photon emission computed tomography/computed tomography shows underlying compression fracture of T-10, T-12

( $n=10)$, Positron emission tomography (PET)/CT $(n=6)]$, musculoskeletal pain $(n=37)$, pathological fracture $(n=1)$, neurological symptoms $(n=1)$, hypercalcemia $(n=1)$ or others $(n=14)$. Metastatic lesions were identified in $32(29 \%)$ patients while 78 (71\%) patients had benign, non-aggressive lesions or normal bone scan as shown in the Figure 1.

Among 32 patients with osseous metastases, unifocal lesion $(25 \%)$ was identified in 8 patients: Axial skeleton $(n=2)$ appendicular skeleton $(n=6) .24$ out of these $32(75 \%)$ patients, had multifocal lesions; axial skeleton $(n=6)$, appendicular skeleton $(n=6)$ and both axial + appendicular $(n=12)$ as shown in the Figure 2. Only $4(12 \%)$ patients had concurrent visceral metastases. In our cohort, based on the location of primary tumour, the frequencies of osseous metastases were; oesophagus $35 \%$ ( 15 out of 43 ), gastric $39 \%$ (7 out of 18 ), gastroesophageal junction $1.5 \%$ (1 out of 8 ) and colorectal $22.5 \%$ ( 9 out of 40 ).

SPECT/CT was acquired in 29 out of 110 patients, which characterized metastatic lesions $(n=12)$ and benign looking non-aggressive entities $(n=17)$. As it is an established fact that SPECT/CT increases the specificity of bone scan [Figures 3-5].

Overall, bone scan upstaged disease in 31\% $(n=34)$ and down staged the disease in $15 \%(n=17)$ patients. 




Figure 5: 50-year-old male with Squamous cell carcinoma of esophagus underwent bone scintigraphy due to hypercalcemia [ionized calcium=1.95 (Normal range 1.09-1.3)]. On Single-photon emission computed tomography/computed tomography images, there was cortical thinning in the lower part of right femoral shaft. No anatomical abnormality was identified in left femoral shaft. These sites were labelled as metastatic involved sites. However Serum magnesium, phosphorus and parathyroid hormone were within normal limits

\section{Discussion}

\section{Indication for bone scans}

In routine no radiological imaging is done exclusively to look for bone metastases in patients with GI malignancy. This is due to very low incidence of bone metastasis in these patients. ${ }^{[2,3,7,8]}$ Portales et al. reported incidence of $3-5 \%$ therefore, there has to be a very specific indication to order a bone scan. ${ }^{[2]}$

In our data, the major indication for referral was an indeterminate lesion on other radiological imaging that was done for routine staging or follow up re-staging. In $51 \%$ cases, the referral for bone scintigraphy was from suspicious lesion on CT scan followed by $9 \%$ from MRI and 5\% from PET/CT scan. Other than anatomical suspicious lesions there have been multiple clinical symptoms and findings leading to evaluation of skeletal metastatic involvement. In our data major clinical symptom included musculoskeletal pain in 34\% cases. Other findings included pathological fracture, neurological symptoms and hypercalcemia.

Portales et al. described these clinical findings as SREs. In the study SREs occurred in $53.6 \%$ of patients. Similar to our results, they observed $(77.2 \%)$ bone pain being the most frequent clinical sign leading to diagnosis of bone metastases followed by $(5.8 \%)$ neurological signs. ${ }^{[2]}$ Other symptoms including hypercalcemia and pathological fractures remained uncommon in both studies.
Our study showed Metastatic lesions in 32 (29\%) patients while 78 (71\%) patients had benign nonaggressive lesions or normal bone scans. In comparison, Portales et al. reported bone metastases on bone scan in 189 patients (5.5\%). Amongst these patients, 173 (91.5\%) patient's bone metastases were diagnosed by bone scintigraphy. ${ }^{[2]}$

This difference may be explained on the basis of selection bias, since our study was a retrospective analysis of bone scans acquired in GI malignancies. Portales et al. selected the patients of GI malignancies who developed bone metastases.

\section{Extent of skeletal disease}

In our data amongst 32 patients with osseous metastasis unifocal lesion $(25 \%)$ were identified in 8 patients. $24(75 \%)$ patient had multifocal lesions.

$4(12 \%)$ patients had concurrent visceral metastasis along with osseous metastasis. Which means exclusive bone metastases was identified in 28 (88\%) patients. This highlights even with a small percentage of patients developing bone metastases, it has great clinical impact. Metastatic skeletal disease appears more aggressive and multifocality is not uncommon. We report more frequent involvement of appendicular skeleton as compared to axial skeleton. In contrast Kanthan et al. ${ }^{[9]}$ showed metastases in axial skeleton in $65 \%$ of patients and $35 \%$ in appendicular skeleton metastases. ${ }^{[6]}$ 


\section{Role of hybrid imaging}

In developing era of hybrid imaging, the role of radiological imaging plays a pivot role in lesion characterization. As demonstrated in our study, SPECT/CT was acquired in 29 out of 110 patients, which characterized metastatic lesions $(n=12)$ and benign looking non-aggressive entities $(n=17)$.

Overall, bone scan upstaged disease in $31 \%$ and down staged $15 \%$ patients. Guezennec et al. showed 91 equivocal uptakes on whole body scans which were further characterized on SPECT/CT. Note was made of $71.4 \%$ benign findings and $14.3 \%$ suspicious findings and $14.3 \%$ remained equivocal even after SPECT/CT. Therefore they down staged 73.6\% and upstaged $15.1 \%$ amongst their equivocal results. ${ }^{[7]}$

\section{Aggressiveness of skeletal disease}

There are random reports in literature which state the appearance of bone metastases preceding the diagnosis of GI malignancies and absence of GI related symptoms. ${ }^{[5]}$ In this scenario, the evaluation of skeletal metastasis becomes more crucial and needs to be done in timely manner.

The sequelae of skeletal metastases, particularly pathologic fractures are reported to affect the overall survival of patient with skeletal metastatic diseases. ${ }^{[3]}$

To summarize, bone scanning as a part of staging workup is a very helpful tool in identifying patients with bone metastases. This finding has a great impact in clinical decision making between local control of unifocal metastases versus palliation for multifocal disease. Bone scintigraphy is not meant to be done in all cases of GI malignancies, but needs to be done as early as possible in patients with symptoms.

\section{Conflict of Interest}

The authors declare that they have no conflict of interest.

\section{References}

1. Global Burden of Disease Cancer Collaboration, Fitzmaurice C, Allen C, et al. Global, regional, and national cancer incidence, mortality, years of life lost, years lived with disability, and disability-adjusted life-years for 32 cancer groups, 1990 to 2015: A Systematic analysis for the global burden of disease study. JAMA Oncol 2017;3:524-48.

2. Portales F, Thézenas S, Samalin E, et al. Bone metastases in gastrointestinal cancer. Clin Exp Metastasis 2015;32:7-14.

3. Saad F, Lipton A, Cook R, et al. Pathologic fractures correlate with reduced survival in patients with malignant bone disease. Cancer 2007;110:1860-7.

4. Agarwal MG, Nayak P. Management of skeletal metastases: An orthopaedic surgeon's guide. Indian J Orthop 2015;49:83-100.

5. Ameur WB, Belghali S, Akkari I, et al. Bone metastasis as the first sign of gastric cancer. Pan Afr Med J 2017;28:95.

6. Santini D, Tampellini M, Vincenzi B, et al. Natural history of bone metastasis in colorectal cancer: Final results of a large Italian bone metastases study. Ann Oncol 2012;23:2072-7.

7. Guezennec $\mathrm{C}$, Keromnes $\mathrm{N}$, Robin $\mathrm{P}$, et al. Incremental diagnostic utility of systematic double-bed SPECT/CT for bone scintigraphy in initial staging of cancer patients. Cancer Imaging 2017;17:16.

8. Besbeas S, Stearns MW Jr. Osseous metastases from carcinomas of the colon and rectum. Dis Colon Rectum 1978;21:266-8.

9. Kanthan R, Loewy J, Kanthan SC. Skeletal metastases in colorectal carcinomas: A Saskatchewan profile. Dis Colon Rectum 1999;42:1592-7. 